\title{
New Magnetospheric Results from the SAMPEX Mission
}

\author{
D.N. BAKER ${ }^{1}$, J.B. BLAKE ${ }^{2}$, L.B. CALLIS ${ }^{3}$, J.R. CUMMINGS ${ }^{4}$, D. HOVESTADT ${ }^{5}$, S. \\ KANEKAL $^{6}$, B. KLECKER $^{5}$, R.A. MEWALDT ${ }^{4}$, AND R. NAKAMURA ${ }^{7}$ \\ 1 Laboratory for Atmospheric and Space Physics, University of Colorado, Boulder \\ 2 Aerospace Corp., Los Angeles, CA 90009 \\ 3 NASA/Langley Research Center, Hampton, VA 23681 \\ 4 California Institute of Technology, Pasadena, CA 91125 \\ 5 Max Planck Institute for Extraterrestrial Physics, Garching, Germany \\ ${ }^{6}$ NASA/Goddard Space Flight Center, Greenbelt, MD 20771 \\ 7 Solar Terrestrial Environment Laboratory, Toyokawa, 442, Japan
}

\begin{abstract}
Results are described from energetic particle detectors onboard the Solar, Anomalous, and Magnetospheric Panicle Explorer (SAMPEX) satellite. Electron data are shown for energies E $>400 \mathrm{keV}$ in the outer zone of electron trapping $(L \geq 3)$. The processes by which electrons are accelerated to very high energies ( $\mathrm{E}>1 \mathrm{MeV}$ ) are discussed. Data are sorted according to L-values and are compared with concurrent solar wind and geomagnetic conditions. Data from SAMPEX are also compared to GOES and UARS measurements. It is found that high-speed solar wind streams drive the acceleration and recirculation of electrons throughout the outer zone on time scales of one day (or less). Very high time resolution measurements from SAMPEX show the very sporadic nature of magnetosphere-atmosphere coupling processes.
\end{abstract}

\section{INTRODUCTION}

Relatively few studies have been able to examine the longterm variation of magnetospheric electrons over a broad range of energies and L-values. Gussenhoven et al. [1987] showed the average pattern of $E \geq 1.0 \mathrm{MeV}$ electrons using dosimeters onboard the Defense Meteorological Satellite Program (DMSP) spacecraft at $840 \mathrm{~km}$ altitude; they saw evidence of 27-day recurrences of flux enhancements throughout one year (1984) of their observations. Williams [1966] also found clear evidence of 27-day periodicity in electrons with $E \geq 280 \mathrm{keV}$ and $E \geq 1$ $\mathrm{MeV}$. Recent studies [e.g.. Imhof et al., 1991] have been able to examine several months of low-altitude electron (E>1.0 $\mathrm{MeV}$ ) data and compare this with $\mathrm{L}=6.6$ measurements. Imhof et al. found a good correlation between intense precipitation spikes at low altitude and trapped flux levels at geostationary orbit. Very recently, Imhof et al. [1994] showed that electron fluxes with E>1 MeV track one another very well at low and high altitudes and that such high energy electrons seem to be accelerated on a rapid ( $<1$ day) time scale.

It is of great interest and importance in magnetospheric physics to understand basic particle acceleration and loss mechanisms and to determine the variation time scales for high-energy electrons throughout the Earth's radiation belts. It has been noted that the relativistic electrons could provide a significant coupling mechanism between the magnetosphere and the Earth's middle atmosphere [Baker et al., 1987b; Callis et al., 1991]. Relativistic electrons in the Earth's magnetosphere are also of considerable practical importance because of their deleterious effect on spacecraft subsystems [Gussenhoven et al., 1987; Baker et al., 1994b].

We present here measurements of relativistic electron flux variations as determined with sensitive sensor systems onboard the Solar, Anomalous, and Magnetospheric Particle
Explorer (SAMPEX). Approximately the first two years of data are considered using several different energy thresholds and covering all relevant L-values. The data show abrupt electron enhancements throughout the outer magnetosphere on time scales of order one day. This suggests a coherent global behavior for electron acceleration. It is concluded that the observed large flux enhancements are due to strong solar wind streams impinging on the magnetosphere and these results are indicative of an acceleration mechanism of considerable strength and efficiency operating in the terrestrial magnetosphere.

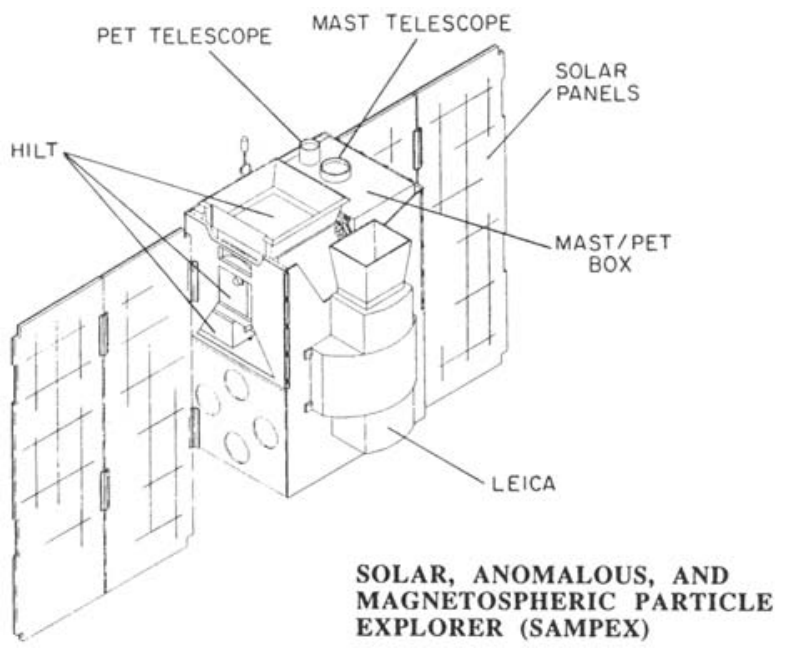

Fig. I The mechanical configuration and layout of the SAMPEX spacecraft showing the positions of the scientific instruments. 


\section{INSTRUMENTS AND DATA ANALYSIS}

The SAMPEX spacecraft was launched from the Westem Test Range at 1419 UT on 3 July 1992 . It achieved the expected altitude of $520 \times 675 \mathrm{~km}$ with an orbital inclination of $81.7^{\circ}$. The SAMPEX payload contains four separate instruments which generally point toward the zenith over the poles of the Earth. The overall configuration of the SAMPEX spacecraft is shown in Figure 1. The orbital pointing strategy and details of the instruments may be found in Baker et al. [1993] and associated papers.

The Proton/Electron Telescope (PET) onboard SAMPEX measures $0.4-30 \mathrm{MeV}$ electrons [see Cook et al., 1993]. One of the PET channels corresponds to a singles rate (P1) from a 2 $\mathrm{mm}$ thick detector which has a threshold of $400 \mathrm{keV}$. (This channel also responds to $>4 \mathrm{MeV}$ protons, but other measurements show that electrons almost always dominate the P1 rate in the outer zone.) Two other coincidence rates in PET (ELO, EHI) correspond to channels that are clearly identified as electrons. ELO responds effectively to 2.1-6.0 MeV electrons and EHI responds to $3.5-15 \mathrm{MeV}$ electrons. There are also several individual singles rates available for the sensors buried deep in the PET stack. PET data are typically averaged over $6-\mathrm{sec}$ in our basic analysis schemes.

The other SAMPEX sensor used in this study is the Heavy Ion Large Telescope (HILT). HILT is a large ion drift chamberproportional counter with solid-state detector (SSD) elements at the back of the telescope [see Klecker et al., 1993]. Although these detectors are also sensitive to $\geq 4 \mathrm{MeV}$ protons, at mid-latirudes the HILT SSDs respond almost exclusively to energetic electrons. Two thresholds ( $\mathrm{E}>1 \mathrm{MeV}$ and $\mathrm{E}>3 \mathrm{MeV}$ ) are set electronically for the HILT SSDs and the 16 individual elements give a very large active area $\left(60 \mathrm{~cm}^{2}\right)$. Considering the wide angle of acceptance for HILT and considering the measured electron detection efficiencies, the HILT $>1 \mathrm{MeV}$ channel has a flux conversion, or effective geometric factor, $\mathrm{g} \sim 100 \mathrm{~cm}^{2}$-sr. The $>3 \mathrm{MeV}$ channel (designated HSSD) has $\mathrm{g} \sim 4 \mathrm{~cm}^{2}-\mathrm{sr}$ and is sampled at $6 \mathrm{sec}$ intervals. The E>1 MeV channel (designated HRSSD) is sampled every $0.1 \mathrm{sec}$.

The PET and HILT telescopes are shown schematically in Figure 2. The data from these instruments are decompressed, dead-time corrected, and converted to absolute intensities. The data are then sorted according to L-value, latitude, longitude, or other appropriate geophysical parameters.

\section{LONG-TERM OBSERVATIONS}

A comprehensive view of selected SAMPEX electron measurements is provided in Figure 3. Here the P1 counting rate data, sorted according to $\mathrm{L}$-value (in bins of $0.1 \mathrm{~L}$ ), are plotted versus day of year (numbered from Day 1 of 1992). The logarithm of the P1 (E>0.4 MeV) electron intensity level in each $\mathrm{L}$ bin has been color-coded according to the reference bar to the right of the figure. L-values ranging from 1.0 to 11.0 are shown. Numerous sharp flux increases occur in the outer zone $(\mathrm{L} \approx 4.0)$ throughout the period shown. Examples of this are seen at many places, but these become particularly prominent after $\sim$ Day 750 . The abrupt enhancements occur in about one

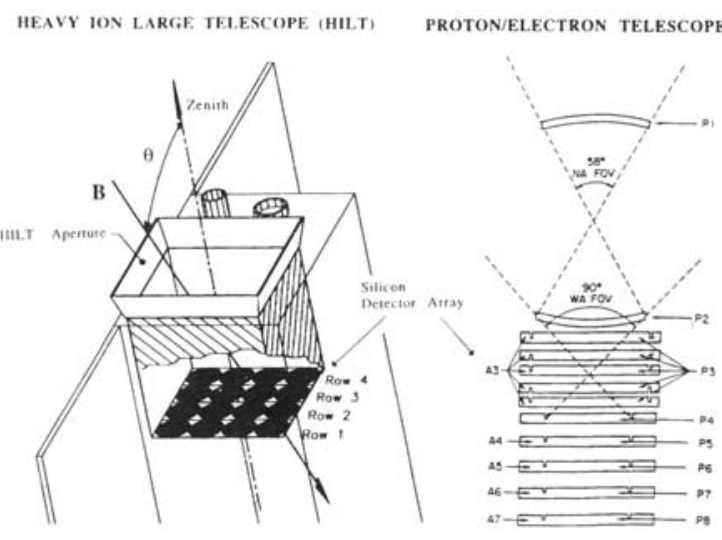

Fig. 2 Cross-section and cutaway diagrams showing the principal detector features of the HILT (left) and PET (right) telescopes of the SAMPEX instrument complement.

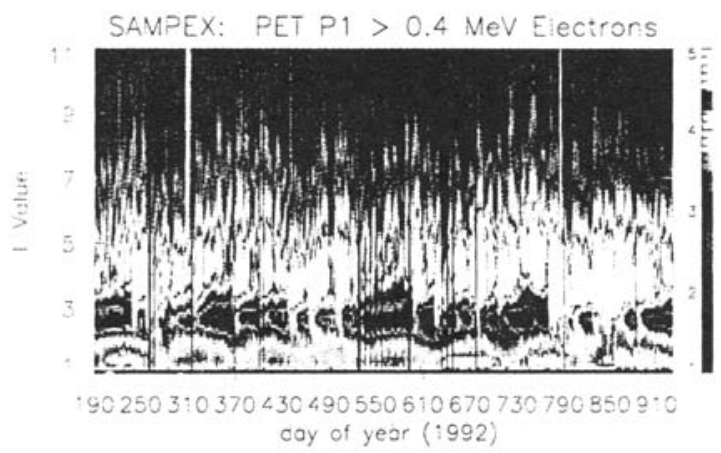

Fig. 3 A color-scale coded representation of electron fluxes measured in the SAMPEX P1 channel (E $\geq 0.4 \mathrm{MeV})$ from July 1992 to July 1994. The data are binned in $0.1 \mathrm{~L}$-values on a daily basis for the range $1 \leq \mathrm{L} \leq$ 11.

See Plate 1a. in the list of color plates.

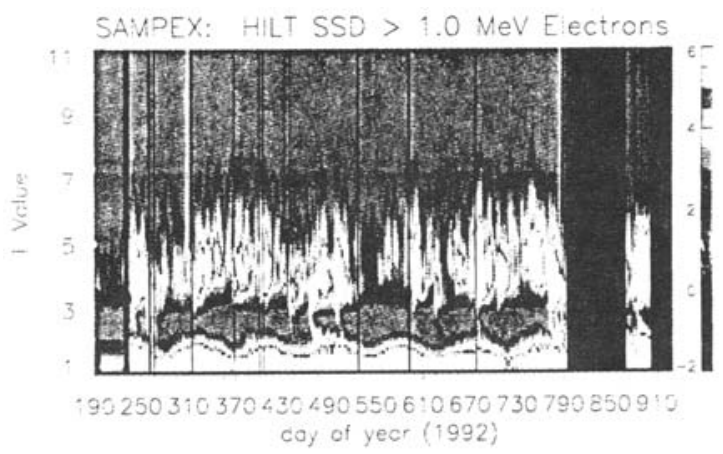

Fig. 4 Similar to Fig. 3 but for the HILT high-resolution channel which measurcs electrons with $\mathrm{E}>1 \mathrm{MeV}$.

See Plate $1 b$. in the list of color plates.

day, extending over a broad range in $L$, evidently reaching to high latitudes and often going beyond $L \approx 6$. These flux increases obviously correspond to the relativistic electron enhancements seen at higher altitudes, e.g., at geostationary orbit [see Baker et al., 1994a]. 
We have also examined both the $>1 \mathrm{MeV}$ and the $>3 \mathrm{MeV}$ channels from HILT using the same L-binning procedure shown for P1. Most of the same features show up in those data. Figure 4, for example, shows the color-coded data for the HILT $>1$ $\mathrm{MeV}$ electrons. The plot shows quite prominently the abrupt enhancements of flux in the outer zone that often occur and also shows several examples of filling in of the region between $L=2$ and $\mathrm{L}=\mathbf{3}$ as was seen in Figure 3 above. (Note that a protective door on the HILT sensor was closed during the period from launch to Day 232 and thus the electrons that penetrated the door and were detected were of much higher incident energy early in the mission.) Unfortunately, the HILT instrument was tumed off between Day 790 and Day 860 to conserve isobutane in the proportional counters. Thus, these data are missing from the record. This is also true for the period Day 900-930.

Note in Figures 3 and 4 that most sharp enhancements at $\mathrm{L} \sim 4.0$ also correspond to abrupt increases extending down to $\mathrm{L} \lesssim$ 3.0. Even the magnetospheric "slot" region around $\mathrm{L} \sim$ 2.5 which separates the inner and outer zones is often temporarily filled with electrons. Previous results [Vampola, 1971] suggested that the slot region is normally filled only during major geomagnetic storms. The SAMPEX data show more frequent effects; significant solar particle events occurred in the period under discussion (see the filled polar cap region after Day 304 and around Day 780), but essentially all other events in the data record are due to high-speed solar wind events. As discussed by Baker et al. [1994a], this means that very frequently the magnetosphere is being "driven" very hard by recurrent solar wind streams.

Inner magnetospheric flux variations in the energy range 2$6 \mathrm{MeV}$ are shown in the line plots presented for several different L-values in Figure 5. The lowest flux trace shows the daily average for $L=2.5( \pm 0.1)$. Typically, this is in the middle of the slot region between the inner and outer electron zone; as a consequence, the flux level is usually rather low in this region. However, for a period around Day 260 the electron flux was very significantly elevated. After Day 300 , the 2-6 MeV electron flux seldom exceeded 1 electron $/ \mathrm{cm}^{2}$-s-sr until Day 770 (i.e., 10 February 1994). At that point the fluxes at $\mathrm{L}=2.5$ rose rapidly and remained high for many months suggesting that the slot region was nearly continuously filled (see Figure 3 also).

The region at $\mathrm{L}=3$ is normally near the inner edge of the outer radiation zone. As seen in Figure 5, this is a highly variable flux region with many large, rapid intensity enhancements that often gradually die away. The $L=3$ trace in Figure 5 shows that there were numerous strong enhancements of $2-6 \mathrm{MeV}$ electrons during 1992-1994, typically occurring every two months or so. However, in February 1994 (i.e., after Day 770) the fluxes jumped up and stayed quite high for several months.

The upper panel of Figure 5 shows the vast difference in electron properties as one goes to the heart of the outer radiation belt at $\mathrm{L}=4$. Here the multi-MeV electron fluxes appear to go up and down by factors of 10-100 on time scales of a few days. (It should be noted that the channel plotted in Figure 5 is subject to high chance coincidences when the rate approaches $10^{4}$; thus, the fluxes near the peak rates in the upper panel are rather uncertain). As seen toward the right of the panel (i.e., beyond $\sim$ Day 600 ), the fluxes are often strongly modulated at $\sim 27$-day intervals. Examination of concurrent solar wind data [e.g., Baker et al., 1994a,b] shows that the peak 2-6 MeV electron fluxes occur a day or two following the time of peak solar wind speeds upstream of the Earth's magnetosphere. Evidently, only the strongest occasional external disturbances affect the region deep in the magnetosphere ( $L 33$ ), while the region further out in the magnetosphere is buffeted much more strongly.

The very outer parts of the Earth's particle trapping regions are subject to strong and frequent disturbances, again due primarily to large solar wind perturbations. If anything, the flux changes at $L \geq 5$ are even more pronounced than those observed at L 4 (as seen in Figure 5 above). In Figure 6, we present a selection of data from the HILT detectors for the period 15 May 1993 to 1 February 1994 (Days 500-763). The data include E>1 $\mathrm{MeV}$ electron fluxes at $\mathrm{L}=5$ (panel (a)), similar data for $\mathrm{L}=6$ (pancl (b)), and electron fluxes for $\mathrm{E}>3 \mathrm{MeV}$ at $\mathrm{L}=6$ (panel (c)).

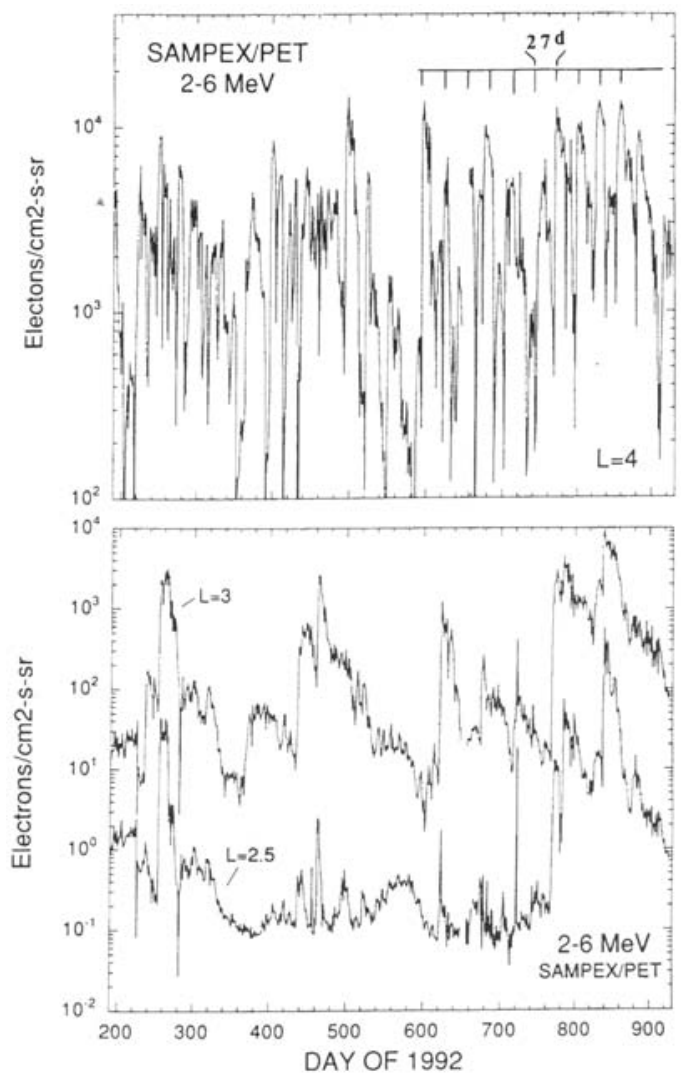

Fig. 5 Daily average fluxes of electrons with $\mathrm{E}=2.1-6 \mathrm{MeV}$ for several selected $L$-values. Data are shown for $L=4( \pm 0.1)$ in the upper panel and for $L=3( \pm 0.1)$ and $L=2.5( \pm 0.1)$ in the lower panel. 
It is evident in Figure 6 that the outer zone electrons measured by HILT exhibit very prominent flux peaks around Days 600,680 , and 750 . As discussed by Baker et al. (1994b), these relativistic electron enhancements were associated with some major, devastating spacecraft operational problems at, or near, geostationary orbit. For example, on 20 January 1994 at 1443 UT, the momentum wheel control circuitry of the Intelsat $K$ spacecraft at geostationary orbit suffered an operational anomaly causing a loss of attitude control. The system was switched to the backup circuitry and control was reestablished. At 1735 UT on 20 January, the Anik E-1 spacecraft, also at geostationary orbit, suffered the same kind of operational anomaly in the momentum wheel control circuitry [Rostoker, 1994]. According to newspaper accounts [e.g., The Hamilton Spectator, 21 January 1994, p. 1]. Telesat Canada operators struggled for 8 hours to regain control of the Anik E-1 satellite. They were able to finally switch to the backup momentum wheel controller and resume reasonably normal operations. Unfortunately, the Anik E-2 satellite also experienced the hard failure of its momentum wheel control circuitry at -0210 UT on 21 January 1994. It was found that the backup circuitry on Anik E-2 was not functional [Rostoker, 1994] and, therefore, it has not been possible to regain full and normal control of the E-2 spacecraft.

The Intelsat and Anik spacecraft provide critical communications functions for Canada. News, weather, and entertainment programming were affected across Canada and daily newspaper information from national news gathering cooperative was interrupted for hundreds of daily papers. Telephone service was intermittent in the northern parts of Canada. Cable television systems, as well as scientific data links such as those associated with CANOPUS, had physically to repoint ground antenna dishes away from Anik E-1 in order to reestablish communication. Thus, television, radio, telephone, and scientific operations were affected for periods of hours to days by these very significant spacecraft anomalies [Baker et al., 1994b].

During the Anik and Intelsat failures, GOES-7 and other geostationary spacecraft showed large enhancements of relativistic electron (E>2 MeV) fluxes [H. Sauer, private comm., 1994; Rostoker, 1994]. The SAMPEX data in Figure 6 support this interpretation. It is quite evident from Figure 6 that the Anik/Intelsat problems occurred when the $\mathrm{E}>1 \mathrm{MeV}$ electrons were near their (recent) historically high values and the same is true of the $\mathrm{E}>3 \mathrm{MeV}$ electrons. Note that the largest peak hux increases in Figure 6 (Day $~ 600,680$, and 750) were also characterized by their long duration (10-15 days). To look specifically near geostationary orbit altitudes, the data in Figure $6 \mathrm{~b}$ and $6 \mathrm{c}$ are for $\mathrm{E}>1 \mathrm{MeV}$ and $\mathrm{E}>3 \mathrm{MeV}$ at $\mathrm{L} \sim 6$. In these plots, the periods near Day 600, Day 680, and Day 750 stand out. In all panels, we have indicated the times of the Anik anomalies.

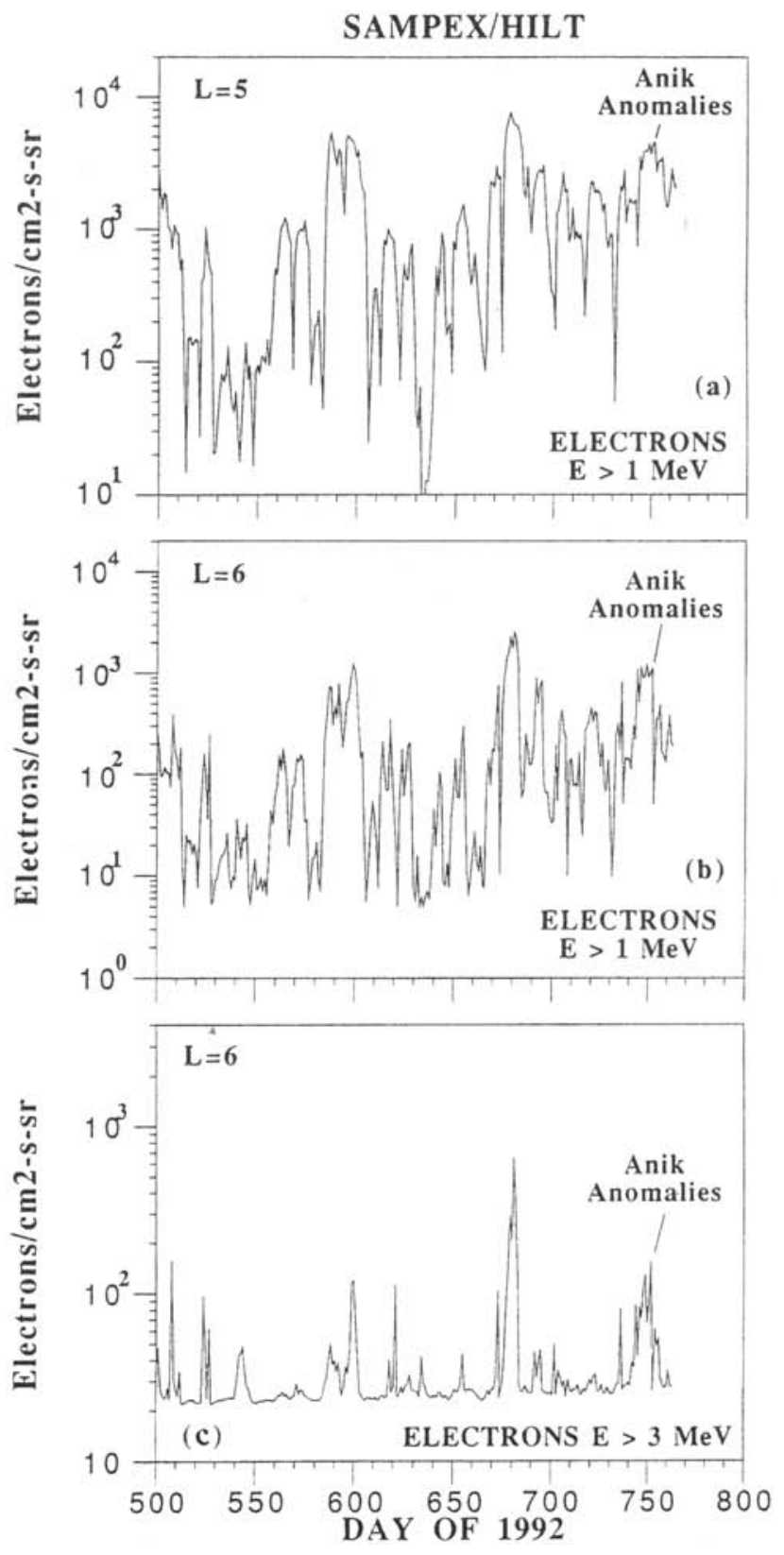

Fig. 6 Daily average of HILT data for Day 500 to Day 763. (a) Electrons with $E>1 \mathrm{MeV}$ for $\mathrm{L}=5$. (b) Electrons with $E>1 \mathrm{MeV}$ for $\mathrm{L}=6$. (c) Electrons with $\mathrm{E}>3 \mathrm{MeV}$ for $\mathrm{L}=6$. 
To illustrate the relationship of electron fluxes to solar wind variations, Figure 7 shows the average daily solar wind speed (top) and the $E>1 \mathrm{MeV}$ electron fluxes ( $L=6$, bottom) for January 1994. The IMP-8 data show a large solar wind streym rising rapidly in speed from January 10-11, 1994, and reaching $\mathrm{V} \sim 750 \mathrm{~km} / \mathrm{s}$. As the speed falls off, the $>1 \mathrm{MeV}$ electrons rise rapidly in absolute intensity. Thus this event is similar to events observed from 1983 to 1985 during the last solar cycle [Baker et al., 1986]. The Anik and Intelsat problems occurred after many days of high electron fluxes.

Early studies of highly relativistic electrons at geostationary orbit [e.g., Paulikas and Blake, 1979] showed strong control by solar wind stream structure; the electron intensities increase markedly a few days after high speed solar wind streams pass over the Earth. Later studies [e.g., Baker et al, 198\% ] showed that large, persistent increases in such electrons were found to be relatively infrequent and sporadic in 1978-81 around solar maximum. During the approach to solar minimum (1981-1986) it was observed that the highly relativistic electrons occur with a regular 27 -day periodicity, and are well associated with solar wind stream structures. Through a superposed epoch analysis technique it was shown that an energetic elecwon enhancement typically rises on a 2- to 3-day time scale and decays on a 3- to 4-day scale at essentially all energies above $\sim 1 \mathrm{MeV}$.

It has been shown [Reagan et al., 1983] that irradiation of space systems by very energetic electrons can cause the deep dielectric charging phenomenon. In this process, very-high. energy electrons can bury themselves in dielectric materials (e.g., coaxial cables, circuit boards, etc.), and stop. They then give rise to very high electric fields (potential differences of several kilovolts) in these regions until eventually an intense breakdown occurs. As noted by Vampola [1987], secondary electrons may be emitted from the surface by incident electrons so that the net charge of the dielectric may remain low. Even so, a large field may build up in the dielectric material itself. Laboratory researchers have modeled the internal conditions within a thick dielectric as it is irradiated with energetic electrons. The potential that results is a function of the flux and energy spectrum of the incident electrons, and of the geometry and conductivity of the dielectric. If the rate of charge deposition by the incident electrons exceeds the rate at which charge leaks out as a result of the conductivity of the material, the potential in the bulk dielectric can exceed the breakdown potential for that material, resulting in a discharge.

Experiments using electron beams to irradiate dielectrics in the laboratory show that breakdown occurs at a typical fluence of $10^{12} \mathrm{e}^{-} / \mathrm{cm}^{2}$, while other studies have examined the response of typical cable and circuit board dielectrics [Vampola, 1987 and references therein]. The laboratory studies suggest that discharges often are rather small and might normally be interpreted as spurious signals. However, these results suggest that discharges produce sufficient energy to damage semiconductors and sensitive devices. As noted by Baker et al. [1987a], most space'bome electronics is becoming increasingly compact and miniaturized; this almost certainly implies an increased sensitivity to damage from deep dielectric discharges.
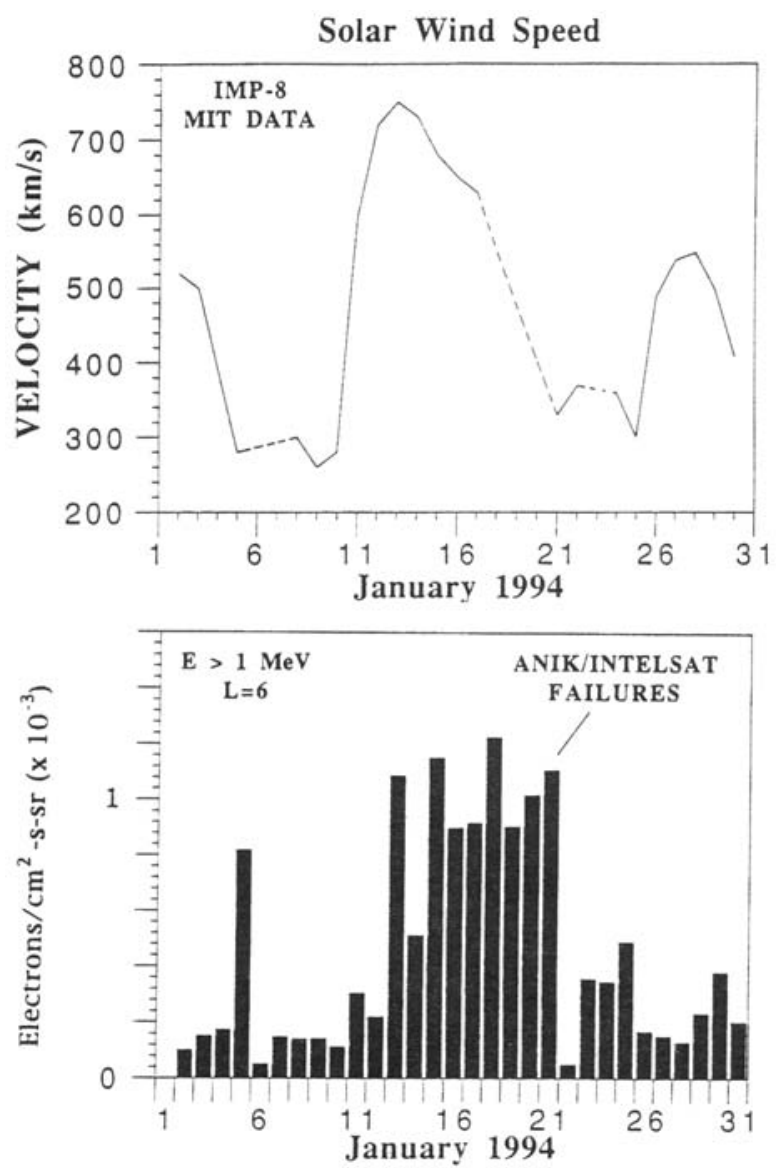

Fig. 7 Detailed solar wind (upper panel) and daily electron fluxes (lower panel) for January 1994 showing conditions related to the Anik satcllite anomalies (from Baker et al., 1994b).

\section{High-RESOlution DATA AND ATMOSPHERIC COUPLING}

SAMPEX data, as shown in the previous section, are very valuable for surveying electron properties throughout the entirety of the radiation belts over long periods of time. From such long-term data, we are able to study electron acceleration and flux decay time scales and we are able to place satellite operational anomalies into a broad space environmental context. Examination of SAMPEX data at higher time resolution, however, reveals exciting new features of electron scattering and loss processes, in particular showing how relativistic electrons couple the magnetosphere to the middle atmosphere.

Over the course of the 11-year solar activity cycle, time-averaged electron radiation levels observed at geostationary orbit have varied by up to $800 \%$ from solar activity maximum to solar activity minimum conditions with peak electron levels occurring during solar minimum conditions [Baker et al., 1987b]. Such radiation variations have been used to estimate the magnitude of the number of electrons penetrating into the atmosphere [Callis et al., 1991]. Knowing these penetrating radiation levels, results derived from an atmospheric model have suggested that large electron variations in the outer magnetosphere may lead to a significant change of global levels of middle-atmo- 
spheric odd nitrogen compounds, and (through complex catalytic cycles) in global ozone [Callis et al., 1991]. The highly inclined $\left(82^{\circ}\right)$ polar orbit, the ability to acquire measurements at all longitudes, and the significant mission lifetime (at least 3 years) provide the SAMPEX mission with an unique opportunity to make direct measurements of the magnetospheric electron radiation and of electrons penetrating into the atmosphere.

Figure 8, for example, shows the pattern of 2-6 MeV electron flux distribution observed by SAMPEX at $\sim 500 \mathrm{~km}$ altitude over the northern hemisphere on Day 254 of 1992 . The greyscale representation of the log of the count rates gives a clear impression of the global distribution of high electron fluxes. The irregular collar of high fluxes seen at midlatitudes is a mapping of the outer radiation belt down onto the upper atmosphere of the Earth. Many of these detected electrons are in the particle loss cones (bounce and drift) and are therefore lost by precipitation into the atmosphere. As consequence, these electrons may cause formation of a variety of nitrogen compounds in the atmosphere which in turn may significantly influence global ozone levels [Callis et al., 1991].

An important aspect of electron coupling from the magnetosphere to the atmosphere is to characterize the incident electron energy spectrum from moderate to relatively high energies. Given the several energy channels available from SAMPEX, and given the high sensitivity of HILT and PET, we are able to derive electron spectra over a broad range. We are also able to compare such spectra with those measured by other concurrently operating spacecraft such as the Upper Atmospheric Research Satellite [UARS; see Gaines et al., 1994].

In Figure 9, we show illustrative spectra derived from PET and HILT data for 10 November 1993. In the extended Day of 1992 scheme, this date (Day 680) was the time of a broad peak in relativistic electron flux throughout the entire outer zone (see Figures 3-6). We also had available for this day some UARS High Energy Particle Spectrometer (HEPS) data for comparison. The data presented in Figure 9 are for $\mathrm{L} 4$, i.e., in the heart of the outer radiation belt. Both trapped and precipitating electron generally would be included in the SAMPEX data since no attempts has been made to include only fluxes measured in the bounce loss cone. However, the loss cone is large $\left(\alpha_{c} \approx 60^{\circ}\right)$ at SAMPEX altitudes implying a significant portion of the sampling by PET and HILT is in the loss cone. On the other hand, the UARS/HEPS data [E. Gaines, private comm., 1994] are from a narrow-angle system looking only at trapped electrons.

It is seen in Figure 9 that the absolute intensities, especially above $-1 \mathrm{MeV}$, ranged very widely near $L=4$ on 10 November. The vertical dashed lines at $0.4,1$, and $3 \mathrm{MeV}$ delineate the highest and lowest integral fluxes seen during the day; excursions of about two orders of magnitude were seen at energies above $\mathrm{E}=3 \mathrm{MeV}$. The UARS/HEPS data available to us were acquired at -0900 UT on 10 November. Those data are plotted in Figure 9 as the solid points. The HEPS integral spectrum is well-fit by an equation of the form $J=K \exp \left[E / E_{0}\right]$ with $E_{O}=$ $0.585 \mathrm{MeV}$. On the other hand, the spectrum derived for SAMPEX (also at $-0900 \mathrm{UT}$ and $\mathrm{L}=4$ ) is much harder with $\mathrm{E}_{0}=$
$0.924 \mathrm{MeV}$. At $E=400 \mathrm{keV}$, the SAMPEX integral flux is about a factor of 2 higher than UARS in this comparison. On the other hand, it should be noted that the UARS/HEPS spectrum does lie within the lower range of integral fluxes seen by SAMPEX, suggesting that there may be reasonable agreement between the data sets when local time and geomagnetic longitude effects are taken into account. We conclude from data such as those shown in Figure 9 that outer zone electron spectra are reasonably well fit using exponential forms. We also find that the spectra are quite hard, often having e-folding energies $\left(E_{0}\right)$ in the range $400-1000 \mathrm{keV}$.

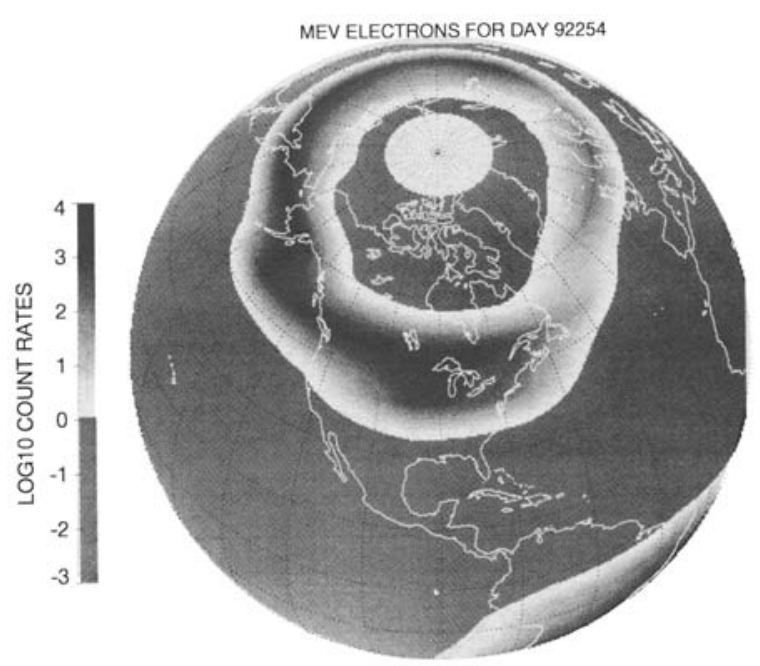

Fig. 8 An image derived from the PET sensor aboard the SAMPEX satellite showing an irregular grey-scaled collar in Northem Hemisphere where very energetic electrons penetrate into the atmosphere. This is a mapping of the Earth's outer radiation belt down to the $500-\mathrm{km}$ altitude of SAMPEX.

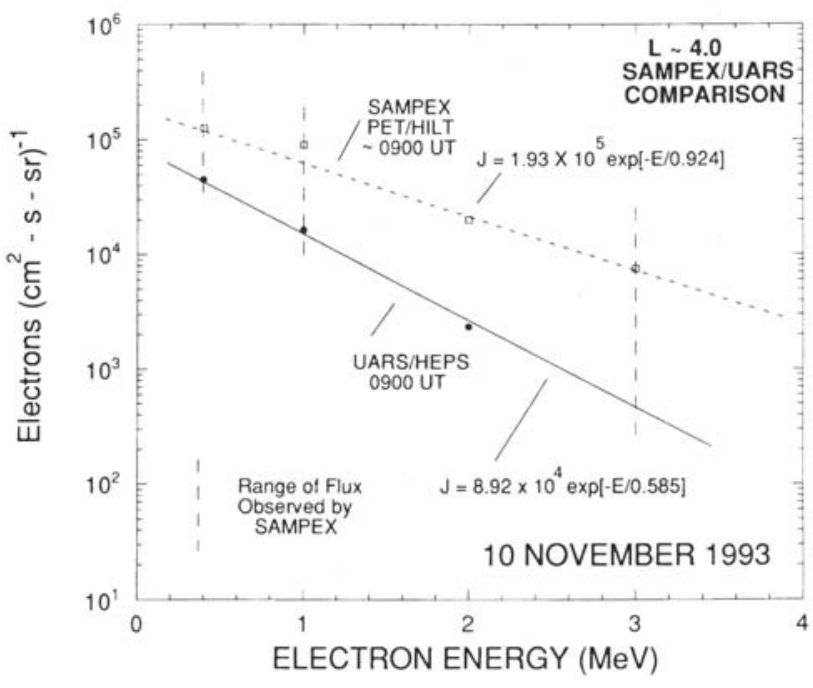

Fig. 9 Integral energy spectra derived from the SAMPEX and UARS spacecraft for 10 November 1993. Exponential spectral forms are fitted to some of the data as described in the text. 
In addition to long-term flux variations and spectral properties of electrons in the outer zone, we have also examined electron intensity changes at very short time scales [Blake et al., 1994; Nakamura et al., 1994]. Broad areas of strong precipitation, extending some $2-3^{\circ}$ in latitude, frequently are observed near the high-latitude boundary of the outer zone. These features can persist for hours and are seen in conjugate locations. A transient form of strong precipitation (microbursts) is also often observed. Microbursts are detected which last for less than a second, indicating that such bursts sometimes occur in a very localized region. The narrow temporal structure is, therefore, a consequence of the orbital velocity of SAMPEX. In other cases, where the spatial size is greater, the temporal evolution of the microburst can be followed in detail [Blake et al., 1994]. These observations clearly indicate that outer-zone electron precipitation frequently results from a strong scattering process, and not by weak diffusion of stably trapped electrons into the drift loss cone [Nakamura et al., 1994].

Figure 10 shows the electron flux (E>1 MeV) from the SSD1 (solid line) and SSD4 (dotted line) sensors of HILT during 3 consecutive crossings of the outer radiation belt in the premidnight sector. The earliest orbit is plotted in the upper panel. The data were obtained by SAMPEX on Day 254 (September 10), 1992 when the satellite was in a pre-midnight - pre-noon orbit (see, also, Figure 8). The subsequent orbits are shown in the middle and lower panels. Each plot starts at the lowest latitude regardless of the satellite direction of motion. The earliest UT shown in each panel is marked at the top. The dashed line at the bottom shows the location of $L=3.7$.

Enhanced fluctuations can be identified mainly at the high latitude portion of the outer radiation belt. During two of the crossings, (0951 and 1022 UT) bands of precipitation with a time scale of $\sim 20 \mathrm{sec}$ were prominent near the high latitude edge of the precipitation region. The flux level of such bands often exceeds that of the main part of the radiation belt. The dotted lines and the solid lines coincide during these $20 \mathrm{sec}$ scale bands, while the two traces slightly differ in the inner region at lower L-values. The SSD1 and SSD4 row of detectors view different local pitch-angles through the HILT aperture. If the electron flux is isotropic over the aperture, the two rows will show identical count rates. If the count rates are not equal, the electron flux is not isotropic. Since HILT is directed toward the zenith, at high latitudes the magnetic field vector is well within the HILT ficld of view and therefore isotropy indicates a filled loss cone. Accordingly, the $20 \mathrm{sec}$-scale bands clearly are located in a region of an isotropic electron population. At lower $L$-values the SSD1 and SSD4 count rates are different, indicating an unfilled loss cone. The 20 sec-scale bands recurrently appear in the region $\mathrm{L}=3.7$ in both hemispheres with a time scale of a few hours, such as in the 0951-1022 UT on this day (and also in the 1305-1442 UT crossings not shown here).

The high degree of variability in observed high-energy electron fluxes, caused both by actual temporal changes and by the rapid motion of a low-altitude satellite through differing magnetospheric regions, has been one of the major difficulties in gaining an understanding of this electron population. One approach in dealing with this problem is to increase the sampling rate of the spacecraft instrumentation. This can lead to severe statistical problems unless there is corresponding increase in the geometric factor of the sensor. The SAMPEX mission contains the kind of instrumentation which combines high-rate sampling of the data with a very large geometric factor, giving a new opportunity to study the precipitation of energetic electrons [Blake et al., 1994].
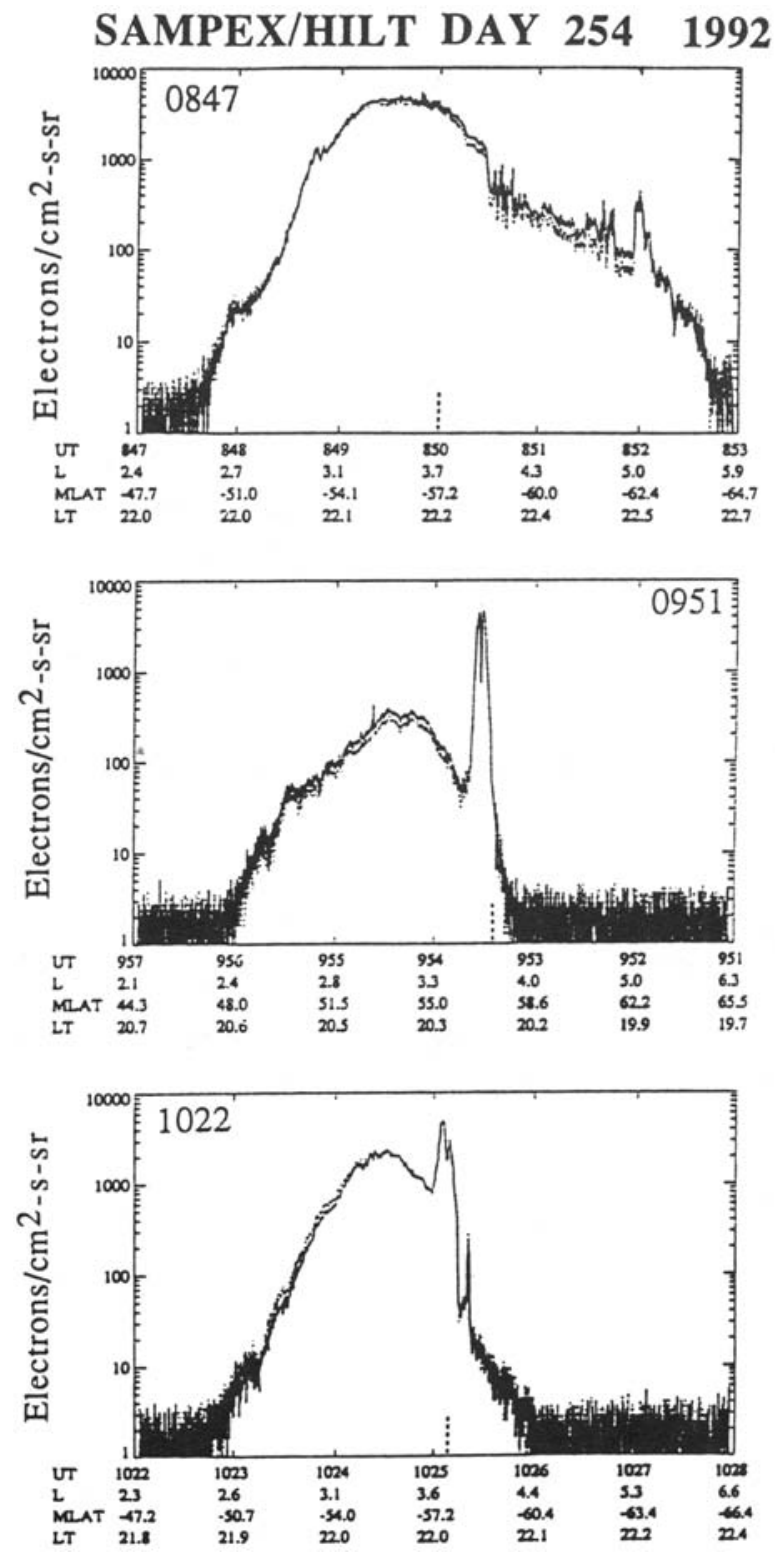

Fig. 10 The electron flux measured by the HILT SSD1 and SSD4 detectors during 3 consecutive crossings of the northem part of the outer radiation belt in the midnight sector on Day 254, 1992 (Adapted from Nakamura et al., 1994). 


\section{Discussion}

A number of previous studies have considered the temporal changes of high-energy electrons at various points in the magnetosphere. For example, Williams [1966] found a 27-day periodicity in the variation of electron fluxes $(E \geq 280 \mathrm{keV}$ and $E$ $\geq 1.2 \mathrm{MeV}$ ) throughout much of the outer zone. He ascribed this variation to the effects of high solar wind kinetic pressures and the concomitant high Alfvenic $M$ ach number $\left(M_{A}{ }^{2}=4 \pi p\right.$ $V^{2} / B^{2}$, where $V=$ solar wind speed, $\rho=$ number density, and $B=$ interplanetary field strength). Later studies examining long-term electron properties have tended to focus on available continuous measurements at geosynchronous orbit [e.g., Paulikas and Blake, 1979; Baker et al., 1986]. These latter authors also found clear evidence of the strong role of high-speed solar wind streams in controlling the intensity of multi-MeV clectrons at $6.6 \mathrm{R}_{\mathrm{E}}$.

Observations presented in this paper reveal that relativistic clectrons increase in absolute intensity, often by a factor of 10 or more, throughout much of the outer magnetosphere on a time scale of order 1 day or less. Abrupt flux enhancements might be expected to occur in the outer magnetosphere, but it is more remarkable that low L-shells, deep within the magnetospheric cavity, would respond so prominently. As noted above, available solar wind data show that the larger electron intensity increases are associated with high-speed solar wind streams impinging upon the magnetosphere.

The present long term survey of energetic electrons suggests that the outer zone can be divided into three regions based upon the rise and decay characteristics of electron fluxes. Between $\mathrm{L}$ $\approx 2$ and $L \approx 4$ the fluxes increase rapidly as the result of a clear geophysical event such as the arrival of a strong solar wind disturbance or shock wave. The loss rate is large and L-dependent. Weeks can pass before a further acceleration event of large magnitude occurs. Between $L \approx 4$ and $L \approx 5.5$ the variations in electron flux are, in general, less dramatic. Enhancements often are less pronounced, but more frequent. Outside $L \approx 5.5$, the intensity changes are relatively large and frequent; they appear often to be driven by recurrent solar wind streams. Major interplanetary events do not seem to be the sole controlling factor as is the case below $\mathrm{L} \approx 4$.

It appears that the region below $\mathrm{L} \approx 4$ is populated by occasional strong acceleration events, corresponding perhaps to a period of greatly enhanced radial diffusion. Presumably the source population is comprised of the abundant population of electrons normally found around $L=4$ and $L=5$. The source "turns off' after a relatively short period and the newly energized electron population decays away at a given location. Because another enhancement may not occur for weeks, a slot region is created as the electron intensities drop very low. Although the electron intensities between $L=4$ and $L=5.5$ wax and wane and the location of the peak intensity varies in radial distance, the source mechanisms are able to maintain a relatively intense energetic electron population.

Given the large, solar wind speed enhancements observed in conjunction with the SAMPEX flux increases, we expect large dynamic pressure $\left(\rho V^{2}\right)$ enhancements. These pressure increases would compress the magnetospheric field substantially. and in so doing would cause time-dependent (and spatiallyvarying) electric fields. However, the high-speed stream effects would certainly not be as strong as a major shock, nor would the compression occur as quickly. We expect for any substantial electric field associated with the changing B-field that electrons from relatively high L-shells would be driven inward by the solar wind stream compression and in the process the relativistic magnetic moment invariant $\left(\mu_{\Gamma}=\mathrm{P} \perp{ }^{2} / 2 \mathrm{~m}_{0} \mathrm{~B}\right)$ would be preserved. Thus, the perpendicular relativistic energy increases in proportion to the square root of the local magnetic field strength (B) at the final particle location. However, it would be surprising in this scenario to achieve very high energization due to the relatively slow compressions associated with solar wind streams. We look forward to a future evaluation of this acceleration mechanism.

Once the electrons are accelerated at a given magnetospheric position, their subsequent behavior at that location is dominated by radial diffusion, pitch angle diffusion, and ultimately atmospheric loss. From the data in Figures 3 and 4, we see some obvious evidence of radial diffusion; such diffusion is manifested by a high-intensity "pulse" of electrons moving gradually toward lower L-values. However, in other cases the time variability of the electron fluxes seems to be dominated by an exponential decay at a given L-value. We interpret this latter feature as corresponding dominantly to an atmospheric loss process after the initial acceleration.

We have shown examples in this paper of strong pitch angle scattering and precipitation of relativistic electrons into the atmosphere on the very local scale. It is a remaining challenge to tie together the precipitation microburst phenomena with the global pattern of electron coupling to the middle atmosphere. Given the evidence for relatively rapid decay of the relativistic electron fluxes, and given the probability that a major effect is loss into the atmosphere, this means that we should be able to analyze quantitatively the global atmospheric source strength for relativistic electron precipitation [see Baker et al., $1987 b$; Callis et al., 1991]. In light of our finding that characteristic electron lifetimes are of order several days in most regions of the magnetosphere, the electron population that is accelerated by passing solar wind streams must represent a strong input source throughout the entire band of atmospheric latitudes that map to the outer radiation zone. Even the inner zone electrons may sometimes contribute significantly. We look forward to a quantitative analysis of this interesting solar-terrestrial linkage issue.

Finally, in this paper we have shown a strong temporal correlation between the occurrence of intense, long-lasting relativistic electron increases in the outer magnetosphere and severe operational anomalies on three nearly identical geostationary communications satellites. We have also discussed the physical mechanism (bulk charging) by which the high energy electrons could produce anomalous behavior in satellite circuitry. We expect to continue to use SAMPEX data to address this kind of very practical and very important space environmental problem.

Acknowledgments. We thank the many individuals who have contributed so extensively to the success of SAMPEX. We especially thank G. Mason, M. Lennard, and D. Hamilton at the 
University of Maryland, E. Stone at Caltech, and R. Boughner at Langley Research Center for major contributions to this research. This work was supported at the U. of Colorado by NASA grant number NAG5-2681. The authors thank W. Imhof, A. Lazarus, and E. Gaines for data and useful discussions on this work.

\section{REFERENCES}

Baker, D. N., J. B. Blake, R. W. Klebesadel, and P. R. Higbie, Highly relativistic electrons in the earth's outer magnetosphere, $\mathrm{I}$. Lifetimes and temporal history 1979-1984, J. Geophys. Res., 91, $4265,1986$.

Baker, D. N., R. D. Belian, P. R. Higbie, R. W. Klebesadel, and J. B. Blake, Deep dielectric charging effects due to high energy electrons in earth's outer magnetosphere, $J$. Electrostatics, 20, 3, $1987 a$.

Baker, D. N., J. B. Blake, D. J. Gomey, P. R. Higbie, R. W. Klebesadel, and $I$. H. King, Highly relativistic magnetospheric electrons: A role in coupling to the middle atmosphere?, Geophys. Res. Lett., I4, 1027, 1987b.

Baker, D. N., G. M. Mason, O. Figueroa, G. Colon, J. Watzin, and R. Aleman, An overview of the SAMPEX mission, IEEE Trans. Geosc., Elec., 31, 531, 1993.

Baker, D. N., J. B. Blake, L. B. Callis, J. R. Cummings, D. Hovestadt, S. Kanekal, B. Klecker, R. A. Mewaldt, and R. D. Zwickl, Relativistic electron acceleration and decay time scales in the inner and outer radiation belts: SAMPEX, Geophys. Res. Lett, 21, 409, $1994 a$.

Baker, D. N., S. Kanekal, J. B. Blake, B. Klecker, and G. Rostoker, Satellite anomalies linked to electron increase in the magnetosphere, EOS, Trans. AGU, 75, 401, $1994 b$.

Blake, J. B., M. D. Looper, D. N. Baker, R. Nakamura, B. Klecker, and D. Hovestadt, New high temporal and spatial resolution measurements by SAMPEX of the precipitation of relativistic electrons, Adv. Space Res., in press, 1994.

Callis, L. B., D. N. Baker, J. B. Blake, J. D. Lambeth, R. E. Boughner, M. Natarajan, R. W. Klebesadel, and D. J. Gorney, Precipitating relativistic electrons: Their long-term effect on stratospheric odd nitrogen levels, J. Geophys. Res., 96, 2939, 1991.

Cook, W. R., et al., PET: A proton/electron telescope for studies of magnetospheric, solar, and galactic particles, IEEE Trans. Geosci. Rem. Sensing, 31, 565, 1993.

Gaines, E. E., D. L. Chenette, W. L. Imhof, C. II. Jackman, and J. D. Winningham, Relativistic electron fluxes in May 1992 and their effect on the middle atmosphere, $J$. Geophys. Res., submitted, 1994.

Gussenhoven, M. S., et al., New low-altitude dose measurements, IEEE Trans. Nuc. Sci., NS-34_676, 1987.

Imhof, W. L., H. D. Voss, J. Mobilia, D. W. Datlowe, J. P. McGlennon, and D. N. Baker, Relativistic electron enhancements: Simultaneous measurements from synchronous and low altitude satellites, Geophys. Res. Lett., 18, 397, 1991.

Imhof, W. L., E. E. Gaines, J. P. McGlennon, D. N. Baker, G. D. Reeves, and R. D. Belian, Relativistic electron flux comparisons at low and high altitudes with fast ime resolution and broad spatial coverage, J. Geophys. Res., 99, 17,421, 1994.

Klecker, B., D. Hovestedt, M. Scholer, H. Arbinger, M. EnL, H. Kastle. E. Kunneth, P. Laeverenz, E. Seidenschwang, J. B. Blake, N. Katz and D. Mabry, KLLT: A Heavy Ion Lerge ares proponional counter Telescope for soler and anomalows cosmic nys, JEEE Trans. Geosci. Rem. Sensing, 31, 542, 1993.

Nakamura, R., D. N. Baker, J. B. Blake, S. Kanekal, B. Klecker, and D. Hovestad, Relativistic electron precipiution near the outer edge of the radiation bell, Geophys. Res. Lett., submitted, 1994.

Paulikas, G. A., and J. B. Blake, Effects of the solns wind on magnetospheric dynamics: Energetic electrons at the synchronous orbit, in Quantitutive Modeline of Magnetospheric Processes, Geophys. Monograph. Aner. Geophys. Union, 21, 180, 1979.
Reagan, J. B., R. E. Meyerol, E. E. Gainea, R. W. Nightingale, P. C. Filber, and W. L. Imhof, Space charging currents and their effects on spacecrafi systems, IEEE Trans. Electr. Insul., 18, 354, 1983.

Rostoker, G., Commentary on the Anik satellite upsets, Report to Telesal Canada, Canadian Networt for Space Research Repor, January 1994.

Vampola, A. L., Electron pitch angle scatuering in the outer zone during magnetically disturbed times, J. Geophys. Res., 76, 4685, 1971.

Vampola, A. L. The serospace environment at high altitudes and its implications for spacecraft charging and communications, $J$. Electrostat., 20, 21, 1987.

Williams, D. J., A 27-day periodicity in outer zone trapped electron interisities, J. Geophys. Res., 71, 1815, 1966. 